\title{
Design of Current Power Sources for a FFC NMR Apparatus: A Comparison
}

\author{
António Roque ${ }^{1,4}$, Sónia F. Pinto ${ }^{2,4}$, João Santana ${ }^{2,4}$, Duarte Sousa ${ }^{2,4}$, \\ Elmano Margato $^{3,4}$, and José Maia ${ }^{1,4}$ \\ ${ }^{1}$ DEE, Escola Superior de Tecnologia de Setúbal, Campus do IPS, Setúbal, Portugal \\ \{antonio.roque, jose.maia\} @estsetubal.ips.pt \\ ${ }^{2}$ DEEC, Instituto Superior Técnico, Technical University of Lisbon, Lisboa, Portugal \\ \{soniafp, jsantana, duarte. sousa\} @ist.utl.pt \\ ${ }^{3}$ Centro de Electrotecnia e Electrónica Industrial, ISEL, Lisboa, Portugal \\ efmargato@isel.ipl.pt \\ ${ }^{4}$ CIEEE - Center for Innovation in Electrical and Energy Engineering, Lisboa, Portugal
}

\begin{abstract}
The power supply of a Fast Field Cycling Nuclear Magnetic Resonance apparatus is typically a current power source, with characteristics that are not fulfilled by most of the commercially available power supplies. This current source is used to supply a specially designed magnet, and should be able to drive a cycling current with a slew rate lower than $3 \mathrm{~A} / \mathrm{ms}$. In this paper, two solutions for this current source are designed and discussed. The first uses two power supplies: the main voltage source and an auxiliary power supply to guarantee the current transitions from the low level to the high level. In the second solution the auxiliary power supply is replaced by a capacitor. To guarantee the required current slew rates, the possibility of using resistors to dissipate the energy stored in the magnet is also discussed.
\end{abstract}

Keywords: current power source, fast field cycling nuclear magnetic resonance, fast transients.

\section{Introduction}

Fast Field Cycling (FFC) Nuclear Magnetic Resonance (NMR) is an experimental and powerful technique used to study the molecular dynamics of different types of compounds [1], [2]. A FFC NMR apparatus includes different modules, as represented in Fig. 1. In the last decades, the performance of this equipment has improved, incorporating the most up-to-date technological advances, which have resulted in: the optimization of the magnet, providing a magnetic induction with very high homogeneity $\left(\Delta B / B\right.$ lower than $\left.10^{-4}\right)[1]$ - [3]; higher efficiency and higher performance of the current source; higher portability, reducing the size of the equipment. In this work, two solutions for the current source are described and compared, fulfilling the requirements of the FFC NMR equipment.

\section{Contribution to Value Creation}

FFC NMR is a powerful technique that owes its advances to the use of new power semiconductors, new materials, new optimization techniques and computational tools. 


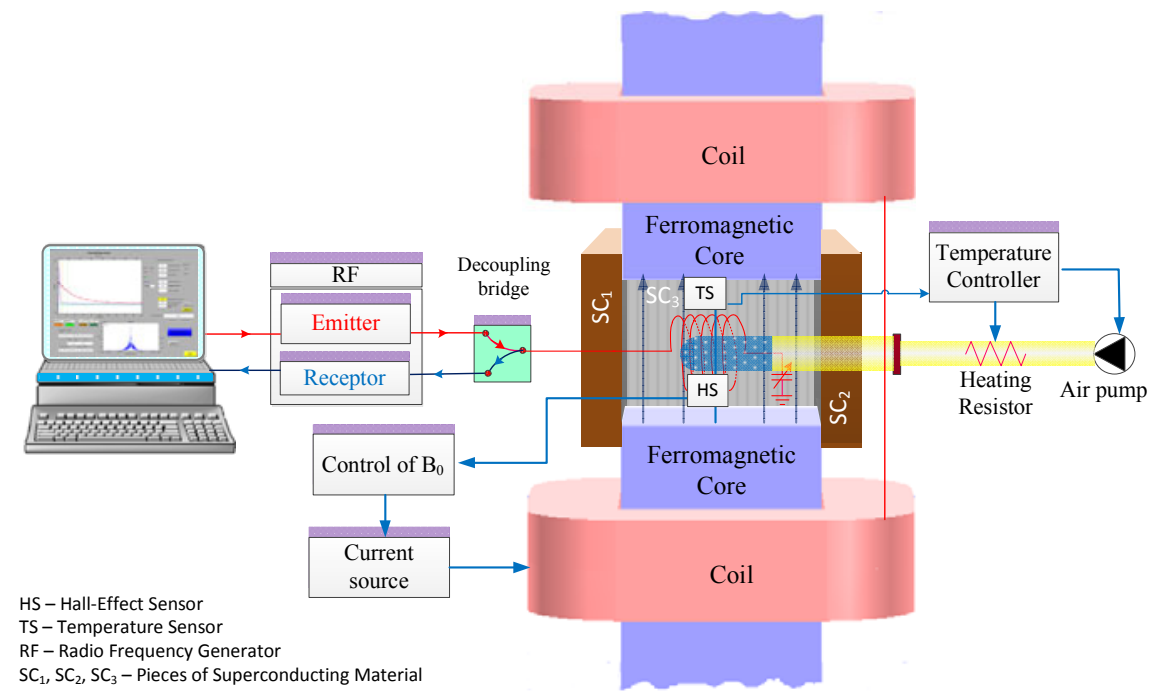

Fig. 1. Main parts of a FFC NMR apparatus

During the last decades the use of FFC relaxometers has been mainly confined to academic specialized laboratories in part due to the fact that these equipments were not commercially available. This has been changing recently and the use of FFC relaxometry has extended to other areas of research, not only in academy but also in industry. This work contributes to value creation, as the development of FFC NMR technology, allowing higher efficiency and performance of the current source, as well as its higher portability and low size will be a factor to spread the usage of this technology in industrial quality control and product development laboratories.

\section{FFC NMR Power Supply}

The power supply of the FFC NMR apparatus is basically a current source that cycles with tunable response times in the ms range. The power supply should allow fast switching of the magnetic field between different values with current slew rates adjusted to less than $3 \mathrm{~A} / \mathrm{ms}$ [4] - [7].

The typical behavior of the magnet current dynamics is represented in Fig. 2, where four different operation modes can be identified:

A: current transition from a low level $\left(I_{\text {low }}\right)$ to a high level $\left(I_{\text {high }}+\Delta I / 2\right)$;

B: current high level or low level (with negative time derivative), bounded by a predefined error window $(\Delta I)$;

C: current high level or low level (with positive time derivative), bounded by a predefined error window $(\Delta l)$;

D: current transition from a high level $\left(I_{\text {high }}\right)$ to a low level $\left(I_{\text {low }}\right)$. 
To achieve these requirements with an adequate efficiency, the FFC current sources are generically designed using two power supplies [1], [3]: a power supply driving the steady state current (main power supply); and a fast-response power supply driving the magnet current during the step up current transients (auxiliary power supply). Several solutions have been developed for each one of these power supplies [3], and therefore, to the global topology of the current source.

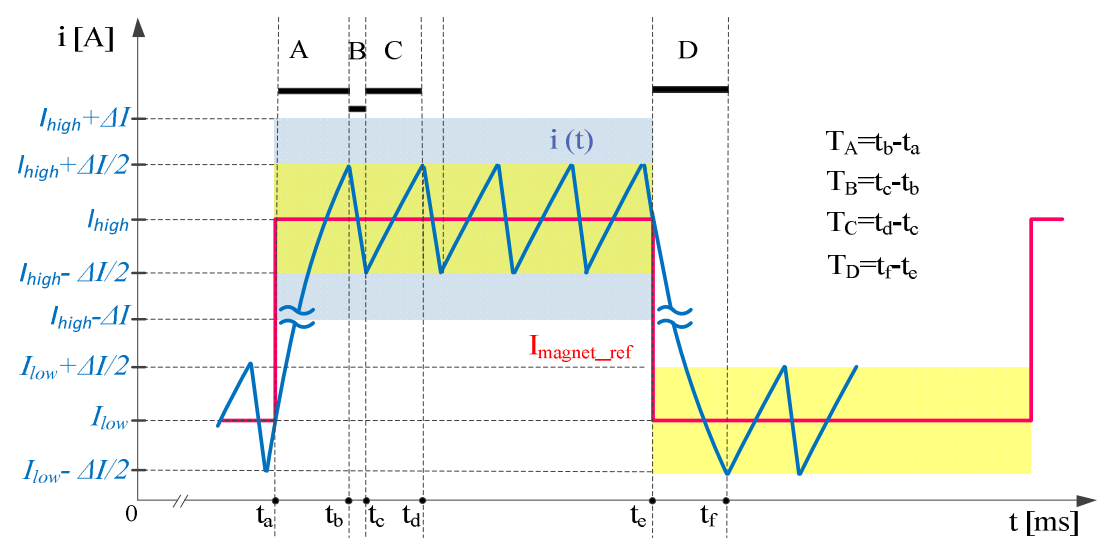

Fig. 2. Typical current waveform of a FFC NMR

In this paper, two possible topologies fulfilling the requirements of this application are presented and compared. For both solutions, the main power supply is based on the topology of a chopper converter. However, the first topology requires an additional dc voltage power supply (based on a full bridge rectifier) to drive the current step up, and includes a damping resistor to guarantee the required current slew rate when the current goes down. In the second topology, a capacitor is used instead of the additional dc power supply, storing energy when the current goes down, and using it to supply the magnet when the current steps up.

\subsection{Current Source with Auxiliary Power Supply and Damping Resistor}

The topology of the FFC NMR current source with an auxiliary power supply and a damping resistor is represented in Fig. 2, where $S_{1}$ and $S_{2}$ represent semiconductors with turn-off capability (as MOSFET); $D_{1}$ and $D_{2}$ are diodes; $U_{0}$ represents the voltage of the main power supply; $U_{a u x}$ is the voltage of the auxiliary power supply (high voltage power supply based on a full bridge rectifier); $R_{l}$ is the damping resistor; $L$ is the self-inductance of the magnet and $R$ is its equivalent resistance.

As the magnet is immersed in liquid nitrogen and cools very slowly, it is difficult to guarantee a constant temperature at the copper coils, which will highly affect the resistance value. As a result, the magnet resistance $R$ (Fig. 3) will not be constant. It has been experimentally measured for different temperature values and the results 
show that the equivalent resistance is nearly 8 times higher at ambient temperature $\left(T_{a}=298^{\circ} \mathrm{K}\right.$ and $R_{T a} \approx 3.0 \Omega$ ) than at liquid nitrogen temperature $\left(T_{N}=77^{\circ} \mathrm{K}\right.$ and $R_{T N} \approx$ $0.4 \Omega$ ). These values will be further considered in the simulations.

With the topology of Fig. 3 it is possible to cycle the magnet current, controlling the states of $S_{1}$ and $S_{2}$. The desired current dynamics is achieved using a high voltage auxiliary power supply $U_{a u x}$ to step up the current (Fig. 4a) and a damping resistor to step down the current (Fig. 4b). The current high level and low level are obtained using only the main power supply $U_{0}$ (Fig. 4c) and the damping resistor $R_{l}$ (Fig. $4 \mathrm{~b}$ ).

Defining $\gamma_{k}$ as (1) and considering Fig. 3, the possible values of $\gamma_{1}$ and $\gamma_{2}$ are presented in Table 1.

$$
\gamma_{k}=\left\{\begin{array}{l}
1 \quad \text { if } S_{k} \text { is } O N \\
0 \quad \text { if } S_{k} \text { is } \text { OFF }
\end{array} \quad k=1,2\right.
$$

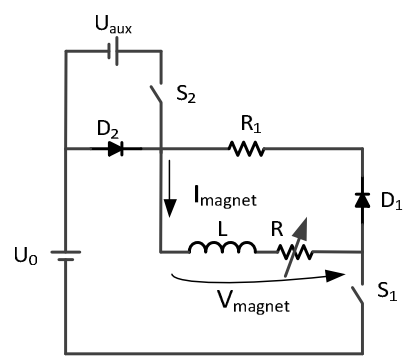

Fig. 3.Topology of the current source with damping resistor

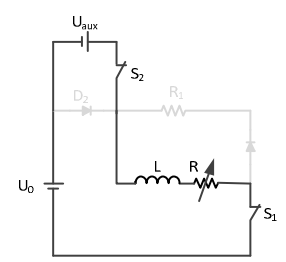

(a) Mode A

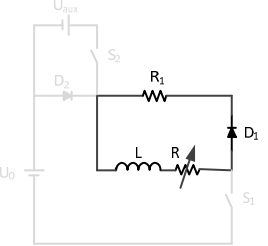

(b) Mode B and D

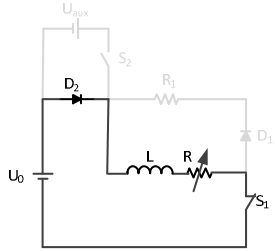

(c) Mode C

Fig. 4. Operating modes of the topology with auxiliary power supply and damping resistor

Table 1. Values of $\gamma_{1}$ and $\gamma_{2}$ for each operating mode of the topology with damping resistor

\begin{tabular}{ccccc}
\hline Topology with damping resistor & $\mathbf{A}$ & $\mathbf{B}$ & $\mathbf{C}$ & $\mathbf{D}$ \\
\hline$\gamma_{1}$ & 1 & 0 & 1 & 0 \\
$\gamma_{2}$ & 1 & 0 & 0 & 0 \\
\hline
\end{tabular}


From (1) and Fig. 2, the equation (2) representing the dynamics of the magnet current is obtained.

$$
\frac{d i_{\text {magnet }}}{d t}=\gamma_{1} \frac{U_{0}}{L}+\gamma_{2} \frac{U_{\text {aux }}}{L}-\left(1-\gamma_{1}\right)\left(1-\gamma_{2}\right) \frac{R_{1}}{L} i_{\text {magnet }}-\frac{R}{L} i_{\text {magnet }}
$$

Equation (2) will be further used in the current controller design.

\subsection{Current Source with Capacitor}

The topology using a capacitor is represented in Fig. 5, where $S_{1}$ and $S_{2}$ are semiconductors with turn-off capability (as MOSFET); $D_{1}$ and $D_{2}$ are diodes; $U_{0}$ is the main power supply voltage; $C$ is the storage capacitor; $L$ is the self-inductance of the magnet and $R$ is its equivalent resistance (variable with temperature).

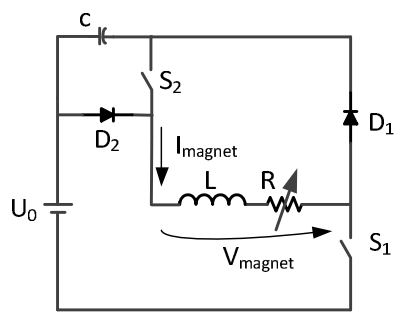

Fig. 5. Topology of the FFC NMR current source with capacitor

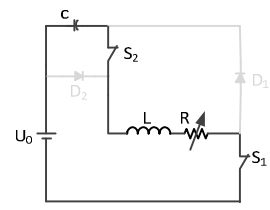

(a) Mode A

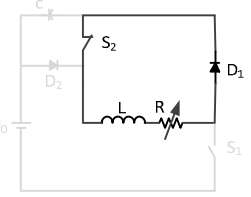

(b) Mode B

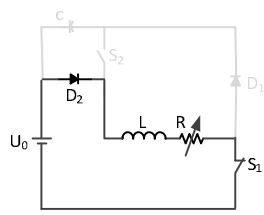

(c) Mode C

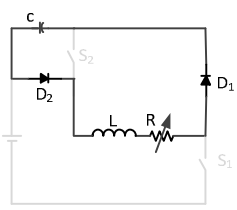

(d) Mode D

Fig. 6. Operating modes for the topology with capacitor

In this topology, the auxiliary power supply of Fig. 3 is replaced by a capacitor, and the damping resistor is not used. The capacitor is then used to store energy when the current goes down (Fig. 6d), and further used to supply the magnet when the current steps up (Fig. 6a). To guarantee the current high level and low level, only the main power supply (Fig. 6c) and free wheeling diode $D_{l}$ are used (Fig. 6b).

From (1), Fig. 5 and Fig. 6, the possible values of $\gamma_{1}$ and $\gamma_{2}$ are as presented in Tab. 2.

Table 2. States of $\gamma_{1}$ and $\gamma_{2}$ for each operating mode of the topology with capacitor

\begin{tabular}{ccccc}
\hline Topology with capacitor & $\mathbf{A}$ & $\mathbf{B}$ & $\mathbf{C}$ & $\mathbf{D}$ \\
\hline$\gamma_{1}$ & 1 & 0 & 1 & 0 \\
$\gamma_{2}$ & 1 & 1 & 0 & 0 \\
\hline
\end{tabular}


From (1) and Fig. 5, it is possible to establish the equations representing the dynamics of the magnet current (3) and the capacitor voltage (4).

From (4), (1) and Fig. 6, the capacitor voltage will change only when both semiconductors $\left(S_{l}\right.$ and $S_{2}$ ) are ON (mode A) or when both are OFF (mode D). If only one semiconductor is $\mathrm{ON}$ (mode $\mathrm{B}$ or $\mathrm{C}$ ), the capacitor charge will remain unchanged.

$$
\begin{aligned}
\frac{d i_{\text {magnet }}}{d t} & =\gamma_{1} \frac{U_{0}}{L}+\gamma_{2} \frac{U_{c}}{L}-\frac{R}{L} i_{\text {magnet }} \\
\frac{d U_{c}}{d t} & =\frac{1}{C}\left(1-\gamma_{1}-\gamma_{2}\right) i_{\text {magnet }}
\end{aligned}
$$

This solution seems clearly more efficient and less expensive than the previous. However it requires a well designed capacitor and a more complex control system.

\subsection{Current Source Controller}

An important requirement of a FFC NMR apparatus is to generate a magnetic induction field with very high homogeneity (magnetic induction field ripple $\Delta B / B$ lower than $10^{-4}$ ) that is directly related to the current ripple. Also, it should guarantee adequate current damping transients. To achieve these requirements, the current control method plays an important role.

For both topologies, a sliding mode controller can be successfully implemented, using hysteretic controllers [7], [8]. To guarantee the current high level $\left(I_{\text {high }}\right)$ and low level $\left(I_{\text {low }}\right)$, operation modes B and C (Fig. 4, Fig. 6), both presenting first order dynamics, are used. Then, the control of the magnet current is ensured by the convergence of the state trajectories to the sliding surface (5) [9], according to the current error, $e_{i}(5)$, where $i$ is the magnet current, $i_{\text {ref }}$ is the reference current and $k$ is a constant $(k>0)$.

$$
S_{e_{i}}=k e_{i}=k\left(i_{\text {magnet_ref }}-i_{\text {magnet }}\right)
$$

To control the magnet current, condition (6) must be guaranteed [9]:

$$
S_{e_{i}} \dot{S}_{e_{i}}<0
$$

The adequate operation mode is chosen from the sliding surface result (5), guaranteeing condition (6):

- If $S_{e_{i}}<0 \Rightarrow e_{i}<0$ and the current should decrease to guarantee $\dot{S}_{e_{i}}>0$ (6).

Then, operation mode B should be chosen for both topologies;

- If $S_{e_{i}}>0 \Rightarrow e_{i}>0$ and the magnet current should increase to guarantee $\dot{S}_{e_{i}}<0$ (6). Then, operation mode $\mathrm{C}$ should be chosen for both topologies. 


\section{Sizing of the Current Power Sources}

To guarantee the adequate operation of both topologies it is necessary to design the damping resistor and the voltage of the auxiliary power supply (topology with damping resistor) and the storage capacitor values (topology with capacitor).

To design the auxiliary power supply it is assumed that the current switches from $I_{\text {low }}$ to $\left(I_{\text {high }}+\Delta I / 2\right)($ mode $\mathrm{A})$ with a slew rate of $2 \mathrm{~ms}$. In this operation mode the current dynamics (7) is obtained from (2), considering $\gamma_{1}=\gamma_{2}=0$ :

$$
\frac{d i_{\text {magnet }}}{d t}=\frac{U_{0}}{L}+\frac{U_{\text {aux }}}{L}-\frac{R}{L} i_{\text {magnet }}
$$

Solving (7), considering Fig. 1 and assuming steady-state operation, the auxiliary power supply voltage may be calculated from (8):

$$
U_{\text {aux }}=\frac{I_{\text {low }} e^{-T_{A} /(L / R)}-R\left(I_{\text {high }}+\Delta I / 2\right)}{e^{-T_{A} /(L / R)}-1}-U_{0}
$$

Considering $T_{A}=2 \mathrm{~ms}, L=270 \mathrm{mH}, R=3 \Omega$ (at ambient temperature), $U_{0}=24 \mathrm{~V}$, $I_{\text {low }}=0.1 \mathrm{~A}, I_{\text {high }}=5 \mathrm{~A}$ and $\Delta I=4 \mathrm{~mA}$, the voltage of the auxiliary power supply is around $U_{a u x}=650 \mathrm{~V}$. To design the damping resistor it is considered the current transient from $t_{e}$ to $t_{f}$ (Fig. 2), where $T_{D}=t_{f}-t_{e}=2 \mathrm{~ms}$. In this operation mode (D) the current dynamics (9) is obtained from (2), considering $\gamma_{1}=\gamma_{2}=0$ :

$$
\frac{d i_{\text {magnet }}}{d t}=-\frac{R_{1}}{L} i_{\text {magnet }}-\frac{R}{L} i_{\text {magnet }}
$$

The damping resistor (10) is calculated solving (9):

$$
R_{1} \approx-\frac{L}{T_{D}} \ln \left(\frac{I_{\text {low }}}{I_{\text {high }}}\right)-R
$$

From (10), assuming $T_{D}=2 \mathrm{~ms}, L=270 \mathrm{mH}, R=3 \Omega$ (at ambient temperature), $I_{\text {low }}=0.1 \mathrm{~A}, I_{\text {high }}=5 \mathrm{~A}$ and $\Delta I=4 \mathrm{~mA}$, the value obtained for the damping resistor is $R_{l} \approx 530 \Omega$. The storage capacitor is designed considering the operating mode $\mathrm{D}$ of the topology with capacitor (Fig. 6). During this transient, it is assumed that the energy stored in the coil is transferred to the storage capacitor, from $t=t_{e}$ to $t=t_{f}$ (Fig. 2) and at $t=t_{e}$ the capacitor is discharged.

$$
\frac{1}{2} L\left(I_{h i g h}{ }^{2}-I_{l o w}{ }^{2}\right) \approx \frac{1}{2} C U_{c}^{2}
$$

From (11), assuming $U_{C}=800 \mathrm{~V}$, the estimated capacity value is $10.7 \mu \mathrm{F}$. 


\section{Simulation Results}

Both topologies were simulated using Matlab/Simulink and considering a variable damping resistor. The main results are presented in Fig. 7 (topology with damping resistor) and Fig. 8 (topology with capacitor). To evaluate the performance of the two power supplies under severe operation conditions, the magnet resistance value is simulated as a sinusoidal waveform, assuming an amplitude of $50 \%$ of its rated value, and a frequency of $8 \mathrm{~Hz}$.

The simulation results of Fig. 7 show that the current cycles, as required by the application, even for severe changes in the magnet resistance. In steady state operation the magnet voltage switches from $24 \mathrm{~V}\left(U_{0}\right)$ to $-2625 \mathrm{~V}\left(R_{I} \times I_{\text {high }}\right)$ or $-52.5 \mathrm{~V}$ $\left(R_{l} \times I_{\text {low }}\right)$, and during the transient from $I_{\text {low }}$ to $I_{\text {high }}$, the magnet voltage is nearly $670 \mathrm{~V}$.
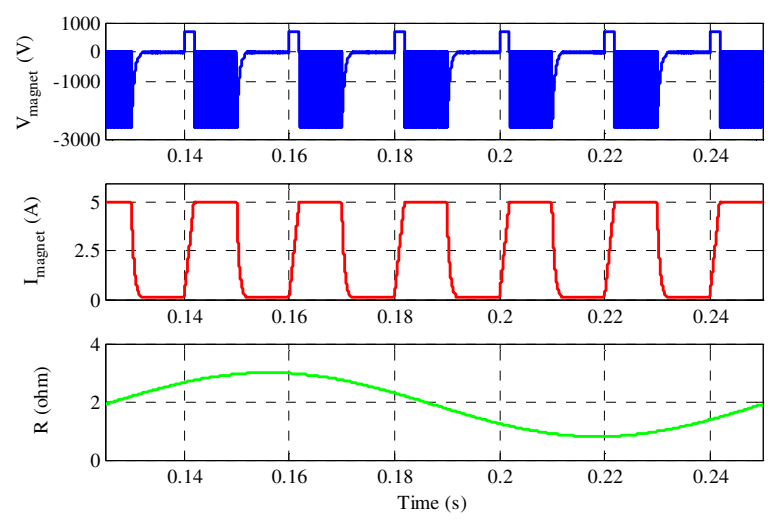

Fig. 7. Steady-state magnet voltage and magnet current for the topology with damping resistor considering a variable magnet resistance

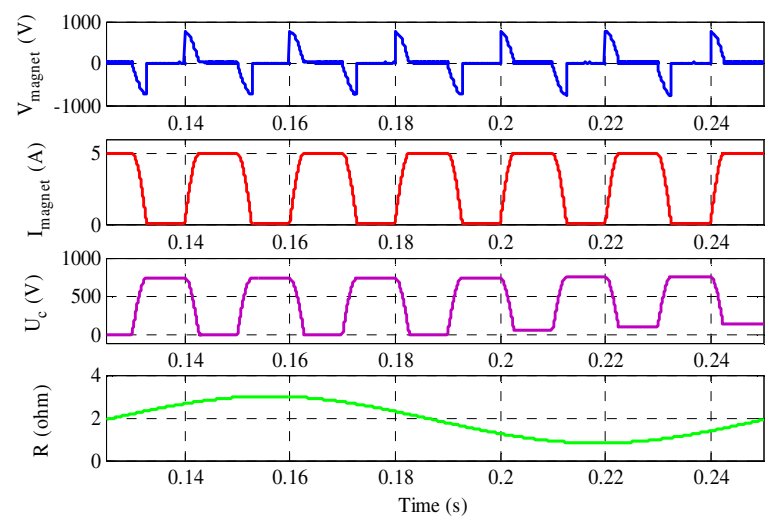

Fig. 8. Steady-state magnet voltage and magnet current for the topology with capacitor considering a variable magnet resistance 
The simulation results of Fig. 8 show that the current cycles are as required by the application, even when the magnet resistance changes. Also, this solution requires an initial charge of the capacitor, which does not affect the FFC NMR experiments. In steady state operation the magnet voltage switches between $24 \mathrm{~V}\left(U_{0}\right)$ and a minimum of $-15 \mathrm{~V}$ (in the case of the maximum current) and during the transients from $I_{\text {low }}$ to $I_{\text {high }}$ or from $I_{\text {high }}$ to $I_{\text {low }}$, it is possible to observe that the magnet voltage equals the storage capacitor voltage.

\section{Conclusions}

In this paper the design and sizing of two topologies for a FFC NMR magnet current source were presented and evaluated. From the simulation results both topologies fulfilled the requirements of the application, allowing the magnet current cycling, as expected. The magnet current ripple is bounded, according to the specifications, and even a 50\% change of the magnet resistance (around its nominal value) did not affect the current controller performances.

Comparing both solutions, the topology with damping resistor is expected to be more expensive due to the additional auxiliary power supply and to the damping resistor. Furthermore, the global efficiency of this solution is also expected to be lower due to the energy losses in the damping resistor. Moreover, the voltage applied to the magnet is higher for a longer period (reaches $2 \mathrm{kV}$ ), which will increase the insulation demand of this setup. In the topology with capacitor a more complex control system is needed and an initial charge of the storage capacitor is required.

Acknowledgements. This work was partially supported by the Portuguese Government, FCT project PEst-OE/EEI/UI4064/2011, CIEEE - "Center for Innovation in Electrical and Energy Engineering" of IST/TULisbon and POSC "Programa Operacional da Sociedade do Conhecimento.

\section{References}

1. Noack, F.: NMR Field-Cyclying Spectroscopy: Principles and Applications. Prog. NMR Spectrosc. 18, 171-276 (1986)

2. Seitter, R., Kimmich, R.: Magnetic Resonance: Relaxometers. Encyclopedia of Spectroscopy and Spectrometry, pp. 2000-2008. Academic Press, London (1999)

3. Anoardo, E., Galli, G., Ferrante, G.: Fast-Field-Cycling NMR: Applications and Instrumentation. Applied Magnetic Resonance 20, 365-404 (2001)

4. Redfield, A.G., Fite, W., Bleich, H.: Precision High Speed Current Regulators for Occasionally Switched Inductive Loads. Review of Scientific Instruments 39, 710 (1968)

5. Constantin, J., Zajicek, J., Brown, F.: Fast Field-Cycling Nuclear Magnetic Resonance Spectrometer. Rev. Sci. Instrum. 67, 2113-2122 (1996) 
6. Sousa, D.M., Fernandes, P.A.L., Marques, G.D., Ribeiro, A.C., Sebastião, P.J.: Novel Pulsed Switched Power Supply for a Fast Field Cycling NMR Spectrometer. Solid State NMR 25, 160-166 (2004)

7. Sousa, D.M., Marques, G.D., Cascais, J.M., Sebastião, P.J.: Desktop Fast-Field Cycling Nuclear Magnetic Resonance Relaxometer. Solid State NMR 38, 36-43 (2010)

8. Bühler, H.: Réglage par Mode de Glissement. Presses Polytechniques Romandes (1986)

9. Silva, J.F.A., Pinto, S.P.F.: Advanced Control of Switching Power Converters, 3rd edn. Power Electronics Handbook. Elsevier (2011) 